\title{
A Highly Potent TACC3 Inhibitor as a Novel Anticancer Drug Candidate
}

Ozge Akbulut', Deniz Lengerli ${ }^{2}$, Ozge Saatci ${ }^{1,3}$, Elif Duman ${ }^{4}$, Urartu O.S. Seker ${ }^{4}$, Aynur Isik $^{5}$, Aytekin Akyol ${ }^{5,6}$, Burcu Caliskan², Erden Banoglu' ${ }^{2}$, and Ozgur Sahin ${ }^{1,3}$

\section{ABSTRACT}

TACC3, a transforming acidic coiled-coil (TACC) family member, is frequently upregulated in a broad spectrum of cancers, including breast cancer. It plays critical roles in protecting microtubule stability and centrosome integrity that is often dysregulated in cancers; therefore, making TACC3 a highly attractive therapeutic target. Here, we identified a new TACC3-targeting chemotype, BO-264, through the screening of in-house compound collection. Direct interaction between BO264 and TACC 3 was validated by using several biochemical methods, including drug affinity responsive target stability, cellular thermal shift assay, and isothermal titration calorimetry. BO-264 demonstrated superior antiproliferative activity to the two currently reported TACC 3 inhibitors, especially in aggressive breast cancer subtypes, basal and HER2+, via spindle assembly checkpoint-dependent mitotic arrest, DNA damage, and apoptosis, while the cytotoxicity against normal breast cells was

\section{Introduction}

Microtubules have been important targets for cancer therapy because of their multifaceted functions in cell division, mitotic spindle formation, intracellular trafficking, and maintenance of cell shape and polarity (1). Microtubule-targeting agents (MTA), such as taxanes, maytansines, vinca alkaloids, colchicine, and combretastatin are therefore commonly used for the treatment of both hematologic and solid malignancies (2). However, the effectiveness of these agents has been impaired by various side effects, such as peripheral neuropathy (3) and possibly by acquisition of resistance to given therapy (4). Mitotic inhibitors, particularly those targeting Aurora kinases and Polo-like kinase 1 (PLK1), have demonstrated promising preclinical results, and several of these are currently being tested in clinics (5). However, the clinical benefit of most of these inhibitors is limited because of low

'Department of Molecular Biology and Genetics, Faculty of Science, Bilkent University, Ankara, Turkey. ${ }^{2}$ Department of Pharmaceutical Chemistry, Faculty of Pharmacy, Gazi University, Ankara, Turkey. ${ }^{3}$ Department of Drug Discovery and Biomedical Sciences, University of South Carolina, Columbia, South Carolina. ${ }^{4}$ UNAM-National Nanotechnology Research Center, Institute of Material Science and Nanotechnology, Bilkent University, Ankara, Turkey. ${ }^{5}$ Hacettepe University Transgenic Animal Technologies Research and Application Center, Ankara, Turkey. ${ }^{6}$ Department of Pathology, Hacettepe University Faculty of Medicine, Ankara, Turkey.

Note: Supplementary data for this article are available at Molecular Cancer Therapeutics Online (http://mct.aacrjournals.org/).

Corresponding Author: Ozgur Sahin, University of South Carolina, 715 Sumter Str., CLS 609D, Columbia, SC, 29208. Phone: 803-777-1891; Fax: 803-777-8356 E-mail: sahin@cop.sc.edu

Mol Cancer Ther 2020;19:1243-54

doi: 10.1158/1535-7163.MCT-19-0957

(C2020 American Association for Cancer Research. negligible. Furthermore, BO-264 significantly decreased centrosomal TACC3 during both mitosis and interphase. BO-264 displayed potent antiproliferative activity $(\sim 90 \%$ have less than $1 \mu \mathrm{mol} / \mathrm{L} \mathrm{GI}_{50}$ value) in the NCI-60 cell line panel compromising of nine different cancer types. Noteworthy, BO-264 significantly inhibited the growth of cells harboring FGFR3-TACC3 fusion, an oncogenic driver in diverse malignancies. Importantly, its oral administration significantly impaired tumor growth in immunocompromised and immunocompetent breast and colon cancer mouse models, and increased survival without any major toxicity. Finally, TACC3 expression has been identified as strong independent prognostic factor in breast cancer and strongly prognostic in several different cancers. Overall, we identified a novel and highly potent TACC3 inhibitor as a novel potential anticancer agent, inducing spindle abnormalities and mitotic cell death.

therapeutic efficacy, necessitating the use of high doses that further leads to unwanted side effects. Therefore, discovery of novel therapeutic targets that are highly essential for mitotic cell division in cancer cells is urgently needed to achieve better antitumor efficacy with lower therapy-related side effects.

TACC 3 is one of the most oncogenic members of the transforming acidic coiled-coil (TACC) family characterized by a conserved Cterminal motif, called the TACC domain that is required for binding to microtubules (6). TACC3 protein localizes at centrosomes and mitotic spindles and may also function as a plus-end tracking protein to regulate microtubule dynamics (7). Its depletion has been reported to inhibit microtubule nucleation at the centrosomes, form multipolar spindles during mitosis upon centrosome declustering (8), and results in chromosome misalignments leading to caspase-dependent apoptosis (9). Moreover, TACC3 inhibition may also reduce mitogenic signaling (10), block epidermal growth factor (EGF)-stimulated epithelial-mesenchymal transition (EMT; ref. 11), and mediate paclitaxel sensitization by triggering post-mitotic $\mathrm{G}_{1}$-phase arrest via activating p53-p21 pathway (12). The mitotic functions of TACC3 are largely controlled by Aurora A kinase, which phosphorylates TACC3 at the Ser558 residue and ensures its recruitment to centrosomes and spindles in association with its interacting partner, ch-TOG (colonic and hepatic tumor overexpressed gene; ref. 13). This is followed by recruitment of clathrin, an endocytic and vesicle-trafficking protein, leading to the formation of the TACC $3 /$ ch-TOG/clathrin complex, which then acts as an intermicrotubule bridge stabilizing kinetochore fibers and preventing microtubule catastrophe (14).

Elevated levels of TACC 3 have been observed in various cancers, including prostate cancer (15), hepatocellular carcinoma (HCC; ref. 16), non-small cell lung cancer (17), and breast cancer (18). Furthermore, downregulation of TACC3 with a small-molecule TACC3 inhibitor, KHS101 (19), reduced tumor growth of glioblastoma xenografts (20). TACC3 inhibition with KHS101 in breast cancer 
cell lines reduced cell viability, motility, EMT, and breast cancer cell stemness and induced apoptotic cell death in vitro (21). Similarly, knockdown of TACC3 suppressed tumorigenesis and cell growth in renal cell carcinoma (22), HCC (16), and other cancers, demonstrating the strong antitumorigenic potential of targeting TACC 3 in cancers overexpressing TACC3. However, there are currently no available TACC 3 inhibitors that have yet reached clinical trials. Encouraged by the well-documented preclinical and clinical implications of TACC 3 in tumor aggressiveness and its strong prognostic power in several different cancers, we directed our efforts to find a novel and potent TACC3 inhibitor and to test its therapeutic potential in different cancers.

Here, we report that we identified a new and potent TACC3 inhibitor, BO-264, through in-house screening of our own chemical library for their ability to inhibit the proliferation of high TACC3expressing JIMT-1 breast cancer cells and verified its interaction with TACC3 using different biochemical approaches. We demonstrated a strong cytotoxic activity of BO-264 at nanomolar levels, which was accompanied by reduced localization of TACC 3 in centrosomes, a strong induction of spindle assembly checkpoint (SAC)-dependent mitotic arrest, apoptosis, and DNA damage. These phenotypes phenocopied the small interfering RNA (siRNA)or short hairpin RNA (shRNA)-mediated knockdowns, demonstrating the on-target specificity of our novel inhibitor. BO-264 has also inhibited the proliferation of cancer cell lines harboring the highly oncogenic FGFR3-TACC3 fusion protein. Most importantly, BO-264 significantly delayed tumor growth in different breast and colon tumor models in a dose-dependent manner with no major toxicity.

\section{Materials and Methods}

\section{Synthesis and analysis of BO-264}

For the synthesis of BO-264, we utilized the synthetic procedure outlined in Supplementary Fig. S1. Briefly, starting from 4-methoxybenzoic acid, ethyl 4-methoxybenzoate (compound 1) was generated through the esterification of the carboxylic acid group. Following the described conditions in Supplementary Fig. S1, 3-(4-methoxyphenyl)3 -oxopropanenitrile (compound 2) was obtained. This $\beta$-ketonitrile intermediate (2) was then treated with hydroxylamine to obtain 3-(4methoxyphenyl) isoxazol-5-amine (compound 3 ), which subsequently underwent a nucleophilic aromatic substitution reaction with 2,4dichloropyrimidine to afford N-(2-chloropyrimidin-4-yl)-3-(4-methoxyphenyl) isoxazol-5-amine (compound 4). Finally, compound 4 was treated with morpholine to obtain the title compound 3-(4-methoxyphenyl)-N-(2-morpholinopyrimidin-4-yl) isoxazol-5-amine (compound 5, BO-264). The experimental details of the synthetic procedures and structure elucidation data are provided in the Supplementary Materials and Methods.

\section{Drug affinity responsive target stability}

Drug affinity responsive target stability (DARTS) was performed as described previously (23). Briefly, JIMT-1 cells were grown to 70\%$80 \%$ confluency and lysed. Cell lysate-drug mixtures were incubated at room temperature on a shaker for 20 minutes to allow binding, and each sample was mixed with $2 \mu \mathrm{L}$ of $8 \mathrm{ng} / \mu \mathrm{L}$ pronase solution (Sigma Aldrich) or buffer only (undigested) and incubated at room temperature. Protein digestion was stopped, and lysates were mixed with $8 \mu \mathrm{L}$ of $4 \times$ SDS loading buffer and heated at $70^{\circ} \mathrm{C}$ for 10 minutes.
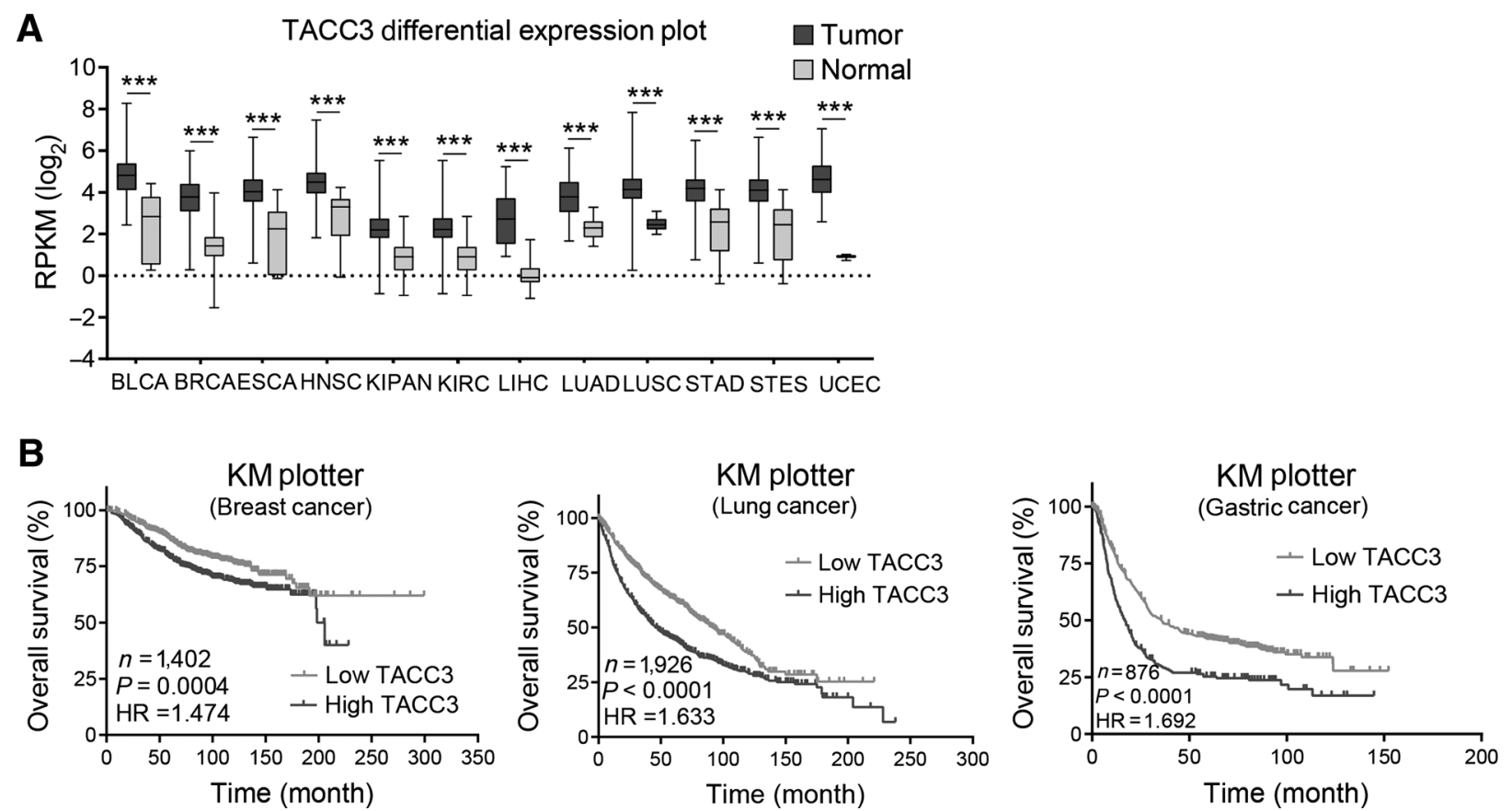

Figure 1.

TACC3 is upregulated in several different cancer types, and its high level is associated with worse overall survival. A, Differential plots of TACC3 between tumor and normal tissues of TCGA patients represented as reads per kilobase million (RPKM; $\log _{2}$ ) values. ${ }^{* * *}, P<0.001$. B, Effect of TACC3 level on overall survival of patients with breast, lung, and gastric cancer retrieved from Kaplan-Meier (KM) plotter database. Log-rank test was used for survival statistical analyses. BLCA, bladder urothelial carcinoma; BRCA, breast invasive carcinoma; ESCA, esophageal carcinoma; HNSC, head and neck squamous cell carcinoma; KIPAN, pan-kidney cohort (KICH+KIRC+KIRP); KIRC, kidney renal clear cell carcinoma; LIHC, liver hepatocellular carcinoma; LAUD, lung adenocarcinoma; LUSC, lung squamous cell carcinoma; STAD, stomach adenocarcinoma; STES, stomach and esophageal carcinoma; UCEC, uterine corpus endometrial carcinoma. 
SDS-PAGE was performed with anti-TACC3 and anti-CDK4 antibody as negative control.

\section{Cellular thermal shift assay}

To analyze the interaction between BO-264 and their target proteins in intact cells, cellular thermal shift assay (CETSA) was performed as described previously with minor changes (24). Briefly, JIMT-1 cells were incubated with vehicle or BO-264 $(1 \mu \mathrm{mol} / \mathrm{L})$ for 6 hours. After treatment, cell pellets were resuspended in TBS buffer containing protease and phosphatase inhibitors. The cell suspension was divided into six PCR tubes and heated for 5 minutes to $45^{\circ} \mathrm{C}, 46^{\circ} \mathrm{C}, 47^{\circ} \mathrm{C}, 48^{\circ} \mathrm{C}, 49^{\circ} \mathrm{C}$, and $50^{\circ} \mathrm{C}$. Subsequently, cells were lysed by three repeated freeze-thaw cycles with liquid nitrogen. Soluble proteins were collected with centrifugation at $20,000 \times g$ for 20 minutes at $4{ }^{\circ} \mathrm{C}$ and analyzed by SDS-PAGE followed by Western blot analysis.

\section{Isothermal titration calorimetry}

Purified TACC3 recombinant protein (TP310754; OriGene) and BO-264 were prepared in $25 \mathrm{mmol} / \mathrm{L}$ Tris $\mathrm{HCl}, \mathrm{pH} 7.3,100 \mathrm{mmol} / \mathrm{L}$ glycine, and $10 \%$ glycerol solution. $\mathrm{BO}-264$ was loaded and titrated with TACC3 protein in duplicate experiments. Titrations were carried out using Microcal 200 Equipment (GE HealthCare) at $25^{\circ} \mathrm{C}$. For each titration, 10 injections were made with 6 minutes spacing. The reference power was set at $2 \mu \mathrm{cal} / \mathrm{second}$, and the sample cell was continuously stirred at $500 \mathrm{rpm}$. The data were analyzed using Origin 7 Software provided along with the ITC200, and binding parameters, such as association constant $(\mathrm{Ka})$, number of binding sites $(N)$, and enthalpy $(\Delta H)$, were calculated.

\section{NCl-60 anticancer drug screening}

BO-264 was submitted to the NCI (NCI number S807620) for screening in the NCI-60 panel of human tumor cell lines. BO-264 was tested twice in the five-dose NCI-60 screen at doses ranging from $10 \mathrm{nmol} / \mathrm{L}$ to $100 \mu \mathrm{mol} / \mathrm{L}$ to determine three endpoints, such as $\mathrm{GI}_{50}$ ( $50 \%$ growth inhibition), total growth inhibition, and lethal dose concentration inducing $50 \%$ cell death values for all 60 cell lines. Briefly, 24 hours after cell seeding into 96 -well plates, cells were treated with $5-\log (\mathrm{mol} / \mathrm{L})$ concentration range of the compound for 2 days. Cytotoxicity was assessed using Sulforhodamine B (SRB) assay. The data presented in the figures are the average values of both experiments.
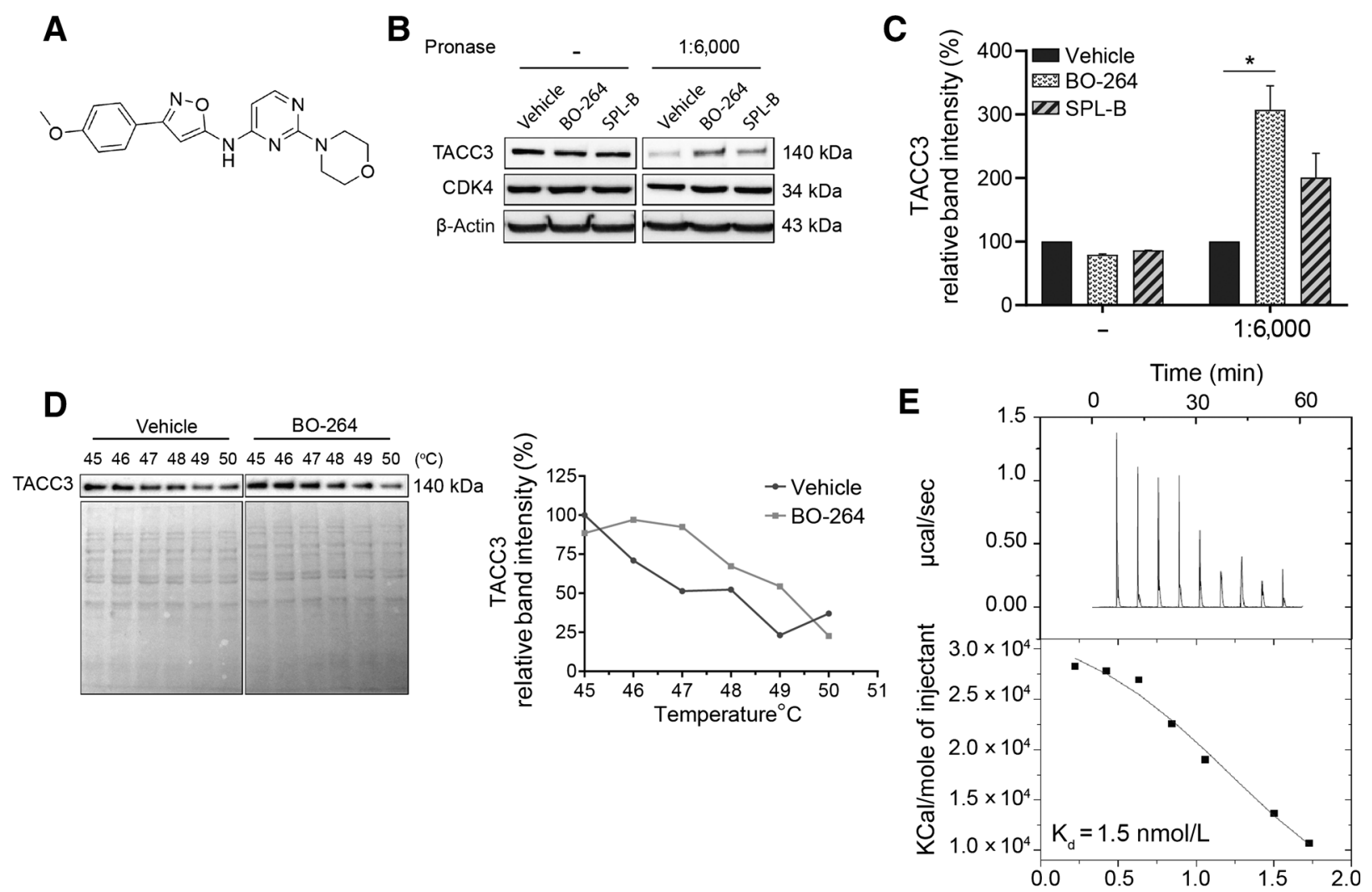

Figure 2.

BO-264 is a novel TACC3 inhibitor binding to TACC3. A, Chemical structure of BO-264. B, Western blot analysis results of DARTS assay using JIMT-1 cell protein extracts to confirm the interaction between BO-264 and TACC3. CDK4 was used as a nontarget protein whose level remains unchanged between treatment groups. Drugs $(10 \mu \mathrm{mol} / \mathrm{L})$ were used. C, Graph showing the quantification of the TACC3 relative band intensity between the treatment groups normalized to $\beta$-Actin and is representative of two independent experiments. Data are presented as means \pm SD. ${ }^{*} P<0.05$. D, Target engagement of BO-264 to TACC3 protein in intact JIMT-1 cells. JIMT-1 cells were treated with vehicle or BO-264 $(1 \mu \mathrm{mol} / \mathrm{L})$ for 6 hours, and cells were collected, heated at the indicated temperatures, and then lysed. Soluble proteins in supernatant were subjected to Western blot analysis, and TACC 3 protein levels were detected. Ponceu staining was used as loading control. CETSA curves indicate TACC3 \% relative band intensity and show the shift between treatment groups. E, Determination of the association constant for the TACC3 and BO-264 interaction using ITC. Top trace shows raw data while bottom trace shows integrated data from a titration of TACC3 into BO-264. Model fitting of a single interaction model was applied using the Origin 7 software provided along with the ITC200 instrument. 
All other methods are provided in Supplementary Materials and Methods.

\section{Results}

TACC3 overexpression is frequent in different cancer types and associated with worse overall survival

To demonstrate the therapeutic potential and the clinical relevance of targeting TACC 3 in cancer, we first analyzed the mRNA expression of TACC3 in different tumor types in comparison with their normal tissue counterparts using The Cancer Genome Atlas (TCGA) data (https://www.cancer.gov/tcga; Fig. 1A). We found that TACC3 is significantly overexpressed in several different tumor types compared with normal tissues, highlighting the potential of TACC 3 as a cancerspecific target. We further analyzed different patient survival datasets using the Kaplan-Meier plotter tool (25) and showed that high TACC3 levels are significantly correlated with worse overall survival in breast, lung, and gastric cancers (Fig. 1B). Clinical relevance to breast cancer has further been validated using an independent breast cancer patient dataset, Molecular Taxonomy of Breast Cancer International Consortium (METABRIC; ref. 26), where we observed a higher TACC3 expression in high-grade tumors (Supplementary Fig. S2A), suggesting a strong correlation with tumor aggressiveness. Importantly, multivariate Cox regression analysis demonstrated that TACC3 expression is an independent prognostic factor in breast cancer (Supplementary Fig. S2B). Altogether, these results suggest that TACC3 is a clinically relevant target and is strongly prognostic in several different cancers, including breast cancer.

\section{BO-264 is identified as a novel TACC3 inhibitor}

Having demonstrated the clinical relevance of targeting TACC3, we sought to identify a novel TACC 3 inhibitor that effectively eliminates cancer cells at nanomolar doses as opposed to currently available TACC3 inhibitors (i.e., SPL-B; ref. 27 and KHS101; ref. 19) working at the micromolar range. To this end, we utilized a strategy to screen the in-house compound collection in which we tested the in vitro cytotoxic potential of the inhibitors followed by functional assays to demonstrate the mechanism of action of our hit compound of interest. In this line, we first performed an in-house screening of a series of small molecules produced in our laboratory to test their effects on cell viability in JIMT1 breast cancer cell line that has a high TACC3 protein expression (Supplementary Fig. S3A). Our screen led to the identification of BO264 (Fig. 2A) as a potential TACC3 inhibitor having an $\mathrm{IC}_{50}$ of 188 $\mathrm{nmol} / \mathrm{L}$. Detailed synthesis of BO-264 is summarized in Supplementary Fig. S1.

To confirm that BO-264 interacts with TACC3, we performed three different protein-drug binding assays; DARTS, CETSA and isothermal titration calorimetry (ITC). DARTS is a label-free strategy to identify potential protein targets for small molecules and is based on stabilization and protection from proteolysis of the target protein upon binding to small molecules $(23,28)$. As shown in Fig. 2B, TACC3 protein was stabilized upon incubation with BO-264 in the presence of the pronase, which was even stronger than that of the known TACC3 inhibitor, SPL-B, which was used as a positive control (ref. 27; Fig. 2B and C). Binding of BO-264 to TACC3 protein has also been validated using the CETSA method, which is based on stabilization and protection of the target protein from aggregation and/or precipitation at

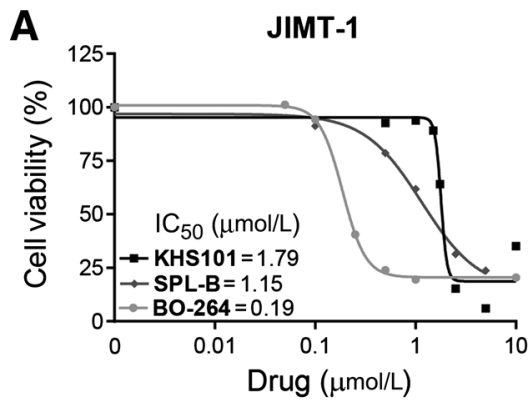

C

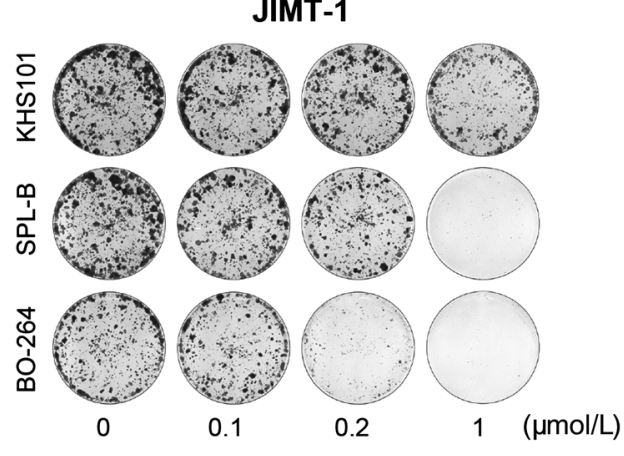

CAL51

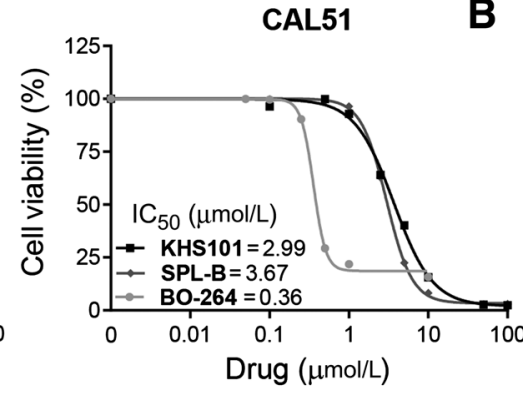

D

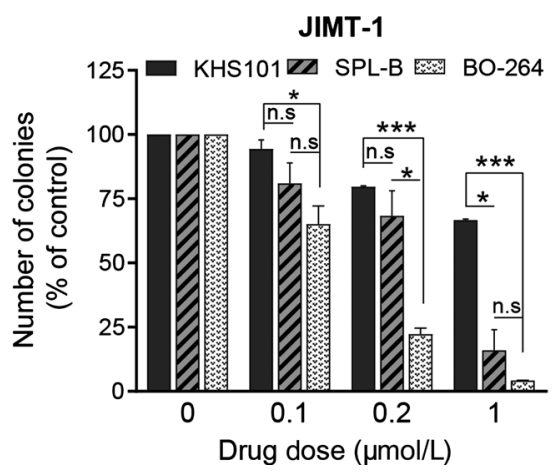

Figure 3.

BO-264 is more effective than other available TACC3 inhibitors, SPL-B and KHS101, specifically targeting cancer cells. A, Pharmacologic inhibition of TACC3 with three different inhibitors and their effect on cell viability of breast cancer cell lines, JIMT-1 and CAL51. Cell viability was measured by SRB assay as triplicates in all the following cell viability experiments, unless otherwise indicated. B, Summary table of the breast cell lines (cancer or normal) tested for responses to three inhibitors. C, Colony formation assay of JIMT-1 cells treated with three inhibitors for 12 days. Colonies were stained with crystal violet. D, Quantification of the colonies was done and analyzed using ImageJ software. Data are presented as means $\pm \mathrm{SD}$ (n.s, not significant; ${ }^{*}, P<0.05 ;{ }^{* * *}, P<0.001$ ). 
high temperatures upon binding to the small molecule (24). As shown in Fig. 2D, treatment of JIMT-1 cells with BO-264 has led to a considerable stabilization of the cellular TACC 3 upon increased temperatures as demonstrated by a shift of thermal melting curve of TACC 3 in the presence of BO-264. As a third method, we employed ITC to directly probe the strength of the physical interaction between BO-264 and TACC3. ITC is a solution-based biophysical approach that is used to determine the thermodynamic properties of a chemical or physical equilibrium (29). A titration of TACC3 protein into BO264 was carried out and monitored by recording the calorimetric and heat changes (Fig. 2E). Subsequent ITC-based binding measurements revealed the interaction between TACC 3 and BO-264 with a dissociation constant, $\mathrm{KD}=1.5 \mathrm{nmol} / \mathrm{L}$ (Fig. $2 \mathrm{E}$ ). Taken together, these data demonstrate that our novel small-molecule inhibitor, BO-264 directly interacts with TACC 3 and therefore acts as a novel TACC3 inhibitor.
BO-264 inhibits the viability of breast cancer cell lines and is superior to currently available TACC 3 inhibitors

Having validated the binding between BO-264 and the TACC3 protein, we analyzed its anticancer potential in comparison with the currently available TACC3 inhibitors, SPL-B (27) and KHS101 (19). To this end, we tested its cytotoxicity in several different breast cancer cell lines, as well as a normal breast cell line, MCF-12A. BO-264 inhibited the viability of basal (MDA-MB-231, MDA-MB-436, CAL51, and HCC1143) and HER2+ (JIMT-1 and HCC1954) breast cancer cell lines at lower doses compared with luminal A (MCF-7, T-47D, and ZR-75-1) and luminal B (BT-474) breast cancer cell lines (Supplementary Fig. S3B). Furthermore, we compared BO$264 \mathrm{IC}_{50}$ with the $\mathrm{IC}_{50}$ of other two TACC 3 inhibitors in most sensitive cell lines and found that BO-264 had a significantly lower $\mathrm{IC}_{50}$ value $(120-360 \mathrm{nmol} / \mathrm{L})$ than SPL-B $(790-3,670 \mathrm{nmol} / \mathrm{L})$ and KHS101 $(1,790-17,400 \mathrm{nmol} / \mathrm{L})$, while no cytotoxic effect of BO-264 and other
A

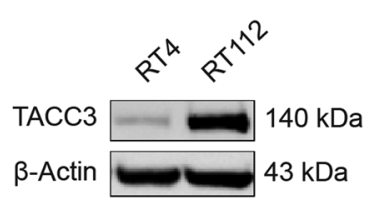

B

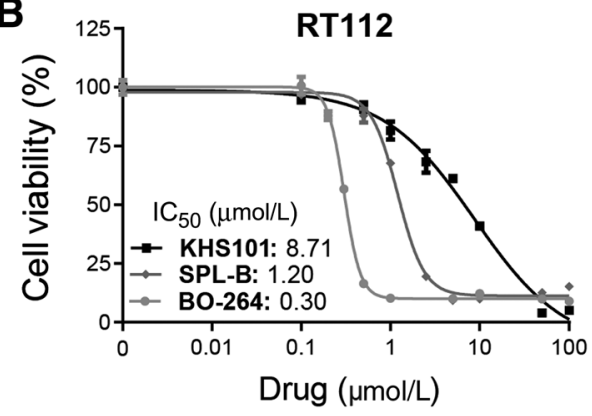

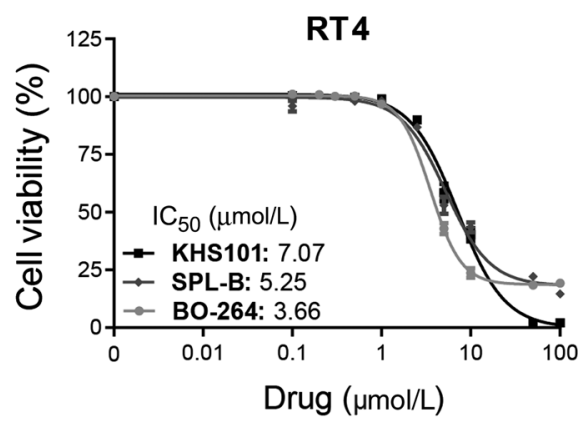

BO-264 SPL-B KHS101

Dose $(\mu \mathrm{mol} / \mathrm{L})-\overline{0.5} \overline{0.5 \quad 5} \overline{0.5 \quad 5}$

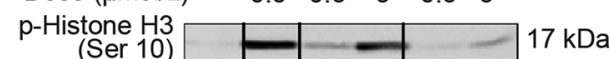

(Ser 10)

p-ERK

(Thr202/Tyr204)

Cleaved PARP

$\beta$-Actin

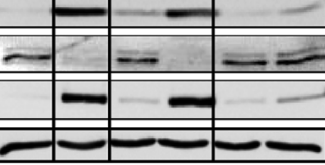

$42 / 44 \mathrm{kDa}$

96 kDa

$43 \mathrm{kDa}$
E

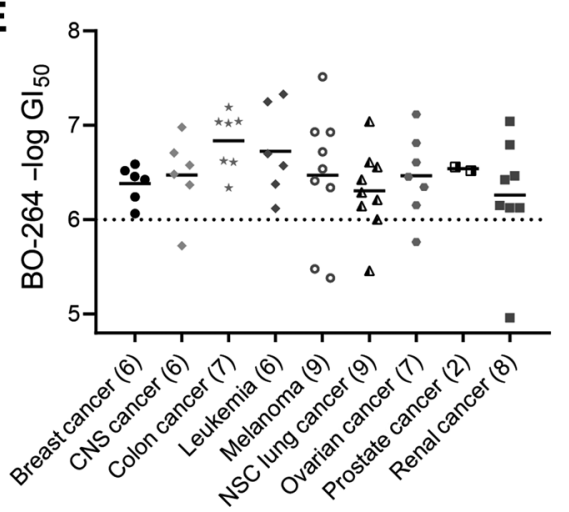

$\mathbf{F}$

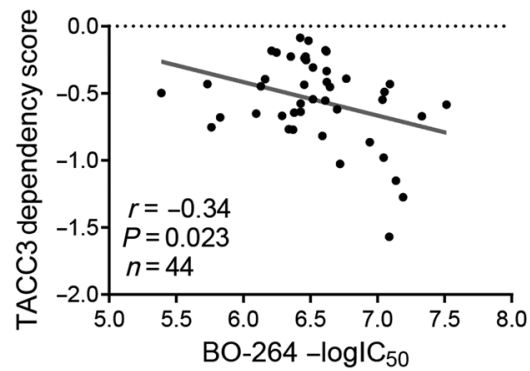

Figure 4.

BO-264 shows remarkable anticancer activity in FGFR3-TACC3 fusion protein-harboring cell lines and in NCI-60 cancer cell lines. A, Western blot analysis of TACC3 protein levels in RT112 and RT4 cells. $\beta$-Actin was used as loading control. B, Pharmacologic inhibition of TACC 3 with three different inhibitors and their effect on cell viability of FGFR3-TACC3 fusion-expressing cell lines, RT112 and RT4. Cell viability was tested upon 3 days of drug incubation and measured by SRB assay. C, Images showing SRB-stained plates after treatment with three inhibitors. D, Western blot analysis of mitotic arrest marker, p-Histone H3, as well as the ERK phosphorylation (p-ERK, Thr202/Tyr204) upon treatment with TACC3 inhibitors at different doses for 24 hours. $\beta$-Actin was used as loading control. E, Average GI 50 values (mol/L) determined from the $\mathrm{NCl}-60$ five-dose screen for BO-264. Black dotted line indicates $1 \mu \mathrm{mol} / \mathrm{L}$ threshold. F, The correlation between $\mathrm{Gl} 50$ values of BO-264 and TACC3 dependency score obtained from DepMap.org. A lower score means that TACC3 is more likely to be dependent in a given cell line. 


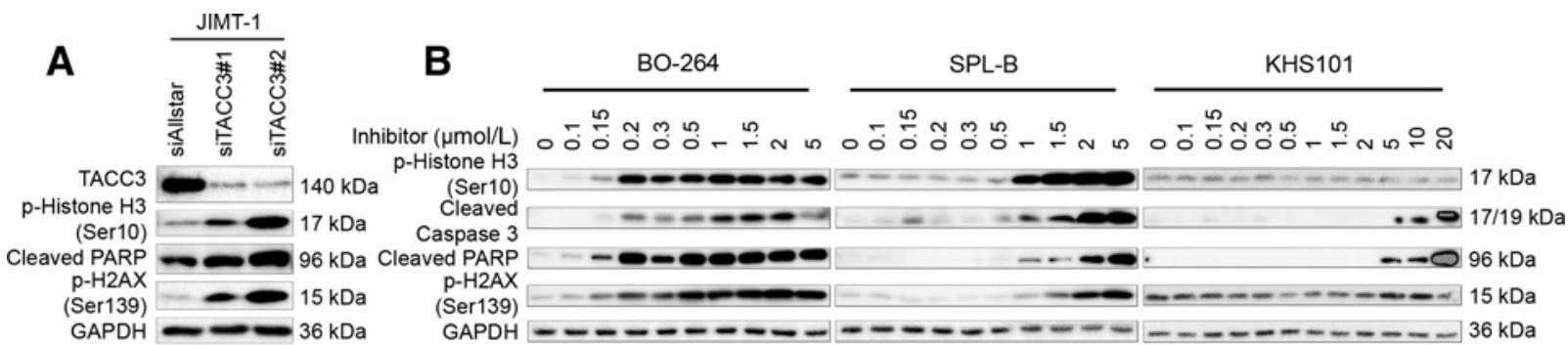

C

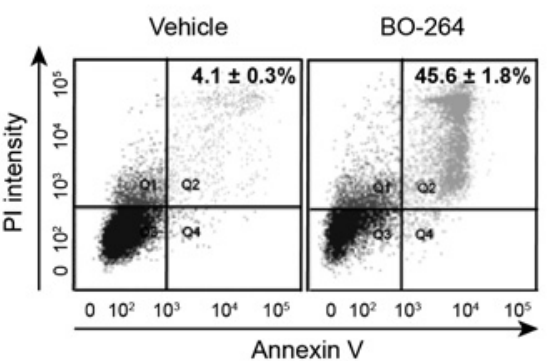

D

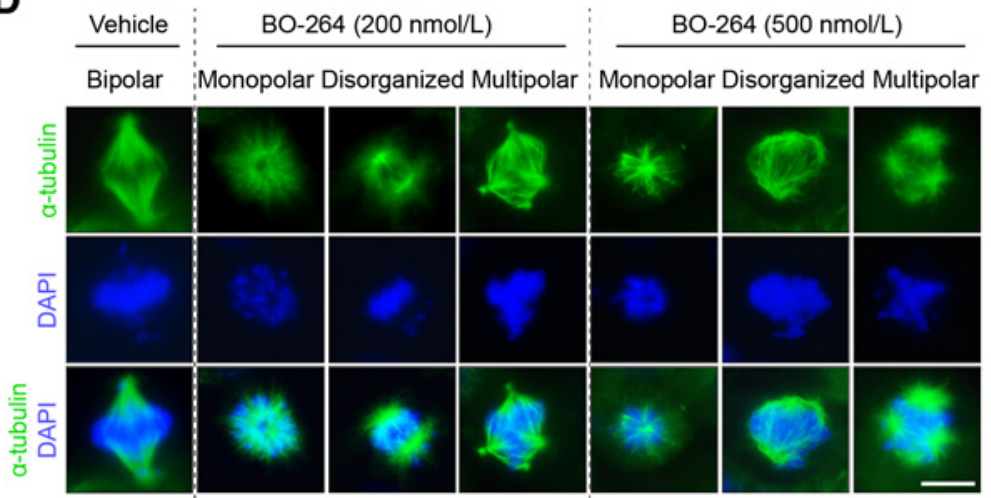

E

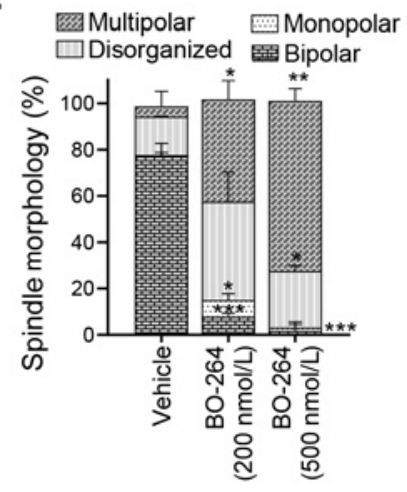

F

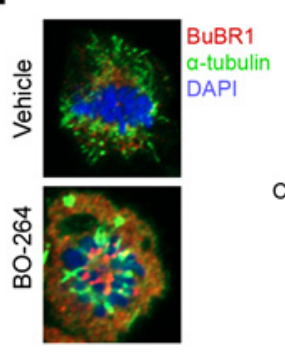

G

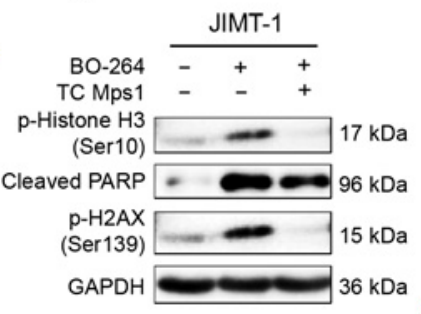

H

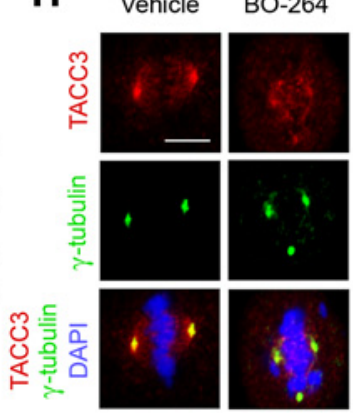

I

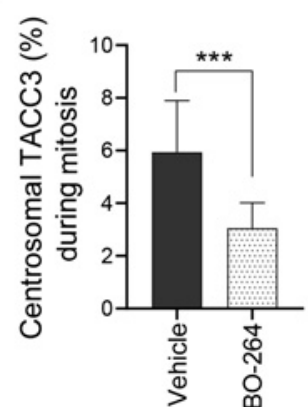

\section{J}

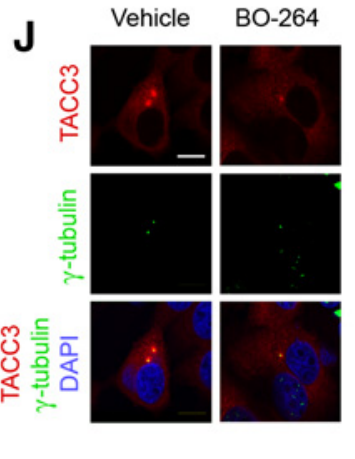

K

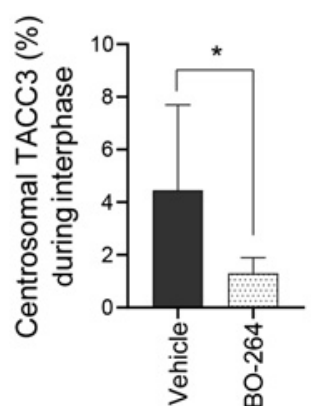

Figure 5.

TACC3 inhibition induces mitotic arrest, apoptosis, and DNA damage and causes aberrant spindles. A, Western blot analysis of mitotic arrest, DNA damage, and apoptosis markers in JIMT-1 cells upon TACC3 knockdown with two different siRNAs. Cells were transfected with 20 nmol/L siRNAs for 48 hours. GAPDH was used as a loading control. B, Western blot analysis of JIMT-1 cells treated with several doses of BO-264, SPL-B, or KHS101 for 24 hours to test the effect on mitotic arrest, DNA damage, and apoptosis markers. The same amount of protein and the same exposure time were used to compare three drugs. C, Annexin V/PI staining of BO-264 (500 nmol/L) treated JIMT-1 cells. The percentage of Annexin V/PI double-positive cells are provided on the graph. D, Induction of aberrant spindle formations in BO-264-treated JIMT-1 cells. Cells were treated with two different doses of BO-264 for 12 hours, fixed in methanol, and then stained with anti- $\alpha$-tubulin (green) antibody. DNA was stained with DAPI (blue). Scale bar, $10 \mu \mathrm{m}$. E, Quantification of spindle abnormalities as seen in $\mathbf{D}$. The data represent the mean values and the SDs of three independent experiments ( $t$ test; ${ }^{*}, P<0.05 ;{ }^{* *}, P<0.01 ;{ }^{* *}, P<0.001$ ). Significance was determined by comparison with vehicle group. (Continued on the following page.) 
TACC3 inhibitors in the normal breast cells was observed (Fig. 3A and B). The superior anticancer activity of BO-264 has also been validated with a colony formation assay using JIMT-1 cells where we demonstrated a significantly lower average colony number of JIMT-1 cells upon treatment with BO-264 (Fig. 3C and D). These results suggest that $\mathrm{BO}-264$ specifically targets breast cancer cells while sparing normal cells.

Next, we analyzed TACC3 expression in cell line models belonging to different subtypes of breast cancer as well as in the normal breast cell line, MCF-12A. As shown in Supplementary Fig. S3A, breast cancer cell lines express higher TACC3 levels than MCF-12A cells, further supporting the cancer-specific high expression of TACC3. Moreover, we observed a trend for increased TACC 3 expression in relatively more aggressive breast cancer subtypes, basal, HER2+, and luminal B as compared with the luminal A cell lines, supporting the notion that TACC3 expression might be associated with cancer aggressiveness. To test the dependency of cells to TACC 3 to survive, we performed a clonogenic growth assay in MCF-12A cells after overexpressing TACC3. As shown in Supplementary Fig. S3C and S3D, TACC3 overexpression increased the cell survival and the colony formation capacity of the normal breast cell line, MCF-12A. Importantly, overexpression of TACC 3 in MCF-12A cells renders them sensitive to the cytotoxic effects of BO-264 that further demonstrates the dependency of cells to TACC 3 and the specificity of our inhibitor (Supplementary Fig. S3E). To exclude the possibility that the differences we observed between cancer and normal cells in terms of BO-264 response are not due to the differences in the proliferation rates of these cells, we calculated their doubling times. As shown in Supplementary Fig. S3F, there was no significant difference between the doubling times of MCF-12A and cancer cell lines, suggesting that responsiveness to BO-264 may not be dictated by increased rates of cell divisions.

\section{BO-264 inhibits the viability of cancer cells with FGFR3-TACC3 fusion and shows very potent cytotoxicity in $\mathrm{NCl}-60$ cancer cell line panel}

FGFR3-TACC3 oncogenic fusion proteins are detected in numerous solid tumors and are emerging as attractive therapeutic targets that will enable selective targeting of fusion-harboring cancers (30). To test whether BO-264 treatment is able to inhibit the growth of fusioncarrying cell lines, we utilized two human urinary bladder cancer cell lines, RT112 and RT4 that are known to harbor FGFR3-TACC3 fusion protein (31). Western blot analysis of TACC3 revealed a higher expression in RT112 cells (Fig. 4A) that was further accompanied by a stronger response to $\mathrm{BO}-264$ as demonstrated by a lower $\mathrm{IC}_{50}$ in RT112 cells (Fig. 4B and C). Despite a relatively lower sensitivity to TACC3 inhibition in RT4 cells (potentially due to lower TACC3 levels), BO-264 yielded the lowest $\mathrm{IC}_{50}$ value in both models as compared with SPL-B and KHS101, suggesting that our novel inhibitor might be a highly relevant therapeutic opportunity to target FGFR3TACC3 fusion-harboring tumors (Fig. 4B). Moreover, BO-264 decreased ERK1/2 phosphorylation, which is a marker for activated FGFR signaling along with a strong mitotic arrest (Fig. 4D) at minimum $10 \times$ lower doses than other TACC 3 inhibitors, suggesting that BO-264 specifically blocks the function of FGFR3-TACC3 fusion protein.

The strong cytotoxic effects that we observed in breast cancer cell lines and the upregulation of TACC 3 in several different cancers further prompted us to test BO-264 in other cancer types. To this end, we performed a dose-response screen of the antiproliferative capacity of BO-264 in the NCI-60 human cancer cell line panel. BO-264 exhibited a remarkable anticancer activity against more than $90 \%$ of the cell lines representing nine different subpanels with $\mathrm{GI}_{50}$ values less than $1 \mu \mathrm{mol} / \mathrm{L}$, further supporting the notion that TACC3 might also be a relevant target in cancers other than breast cancer (Fig. 4E). Notably, we found that $\mathrm{GI}_{50}$ values of NCI-60 cell lines show a positive correlation with the TACC3 dependency score obtained from DepMap.org (ref. 32; Fig. 4F). This indicates that cells which are more dependent on TACC 3 , that is, having low TACC3 dependency score, are more sensitive to BO-264. Collectively, these data demonstrate the strong anticancer activity of BO-264, highlighting its potential applications also in other cancer types.

\section{TACC3 inhibition with BO-264 induces mitotic arrest, apoptosis,} and DNA damage, causes aberrant spindle formation, and reduces centrosomal localization of TACC3

TACC3 depletion in HeLa cells has been shown to cause mitotic arrest (9) and caspase-dependent apoptosis (33). Moreover, we recently showed that depletion of PLK1, a mitotic gene, in T-DM1-resistant breast cancer cells causes mitotic arrest and caspase activation, leading to apoptosis and DNA damage (34). To test whether a similar effect is observed upon TACC3 inhibition in breast cancer, we first did gene set enrichment analyses of mitotic progression and DNA repair-related gene sets in breast cancer patient datasets and showed that mitotic and DNA repair gene sets were significantly enriched among patients with higher levels of TACC3 (Supplementary Fig. S4A). We then used two different siRNAs targeting TACC3, as well as the small-molecule inhibitors, BO-264, SPL-B and KHS101 to test the effects of TACC3 on these processes. Western blot analysis of mitotic arrest, apoptosis, and DNA damage-related markers in JIMT-1 cells revealed a prominent induction of p-Histone $\mathrm{H} 3$ (Ser10) indicating mitotic arrest (35), cleaved PARP indicating apoptosis (36), and p-H2AX (Ser 139) indicating DNA damage (37) upon silencing of TACC3 expression with two different siRNA sequences (Fig. 5A; Supplementary Fig. S4B). Knockdown efficiency of TACC3 was confirmed with qRT-PCR in all breast cancer cell lines tested (Supplementary Fig. S4C). In-line with the activation of mitotic cell death pathways, TACC3 knockdown led to a significant growth inhibition in these cells (Supplementary Fig. S4D), supporting the importance of TACC3 in breast cancer cell survival.

To determine whether similar molecular changes are observed upon TACC3 inhibition with BO-264 and to test its superior activity as compared with available TACC3 inhibitors, we performed Western

(Continued.) F, Immunofluorescence staining of BubR1 (red) and $\alpha$-tubulin (green) in vehicle versus BO-264 (500 nmol/L) treated JIMT-1 cells. G, Western blot analysis of mitotic arrest, DNA damage, and apoptosis markers showing mitotic slippage in BO-264-treated JIMT-1 cells upon treatment with a spindle checkpoint kinase (Mps1) inhibitor, TC Mps1. JIMT-1 cells were treated with $200 \mathrm{nmol} / \mathrm{L}$ BO-264 for 24 hours followed by 12 hours treatment with $1 \mu \mathrm{mol} / \mathrm{L}$ TC Mps1. GAPDH was used as a loading control. H, Immunofluorescence images of JIMT-1 cells during mitosis treated with either vehicle or BO-264 ( 300 nmol/L) for 24 hours, followed by staining with antiTACC3, anti- $\gamma$-tubulin antibody, and DAPI. Scale bar, $10 \mu \mathrm{m}$. I, Quantification of the relative centrosomal localization of TACC3 during mitosis in these treatment groups as seen in H. J, Immunofluorescence images of JIMT-1 cells during interphase treated with either vehicle or BO-264 (300 nmol/L) for 24 hours, followed by staining with antiTACC3, anti- $\gamma$-tubulin antibody, and DAPI. Scale bar, $10 \mu \mathrm{m}$. K, Quantification of the relative centrosomal localization of TACC3 during interphase in these treatment groups as seen in $\mathbf{J}$. ${ }^{*}, P<0.05 ;{ }^{* * *}, P<0.001$. 
blot analysis of mitotic arrest, apoptosis, and DNA damage markers in JIMT-1 cells treated with increasing doses of BO-264 as well as SPL-B and KHS101. As shown in Fig. 5B, siTACC3-induced mitotic arrest, apoptosis, and DNA damage were fully recapitulated by BO-264 in a dose-dependent manner. On the other hand, SPL-B and KHS101 treatments induced the expression of the markers at considerably higher doses as compared with BO-264-treated cells. Furthermore, treatment of JIMT-1 cells with BO-264 for 48 hours induced a prominent increase (from $4.1 \%$ to $45.6 \%$ ) in the fraction of apoptotic cells as assessed by Annexin V/propidium iodide (PI) staining (Fig. 5C). We also knocked down TACC3 with an inducible shRNA and examined mitotic arrest, DNA damage, and apoptosis markers using Western blotting to show that the effect of BO-264 on downstream target is due to TACC3 inhibition, but not due to off-target effects (Supplementary Fig. S4E). Our results showed that the knockdown of TACC3 with an inducible shRNA against TACC3 leads to increased p-Histone $\mathrm{H} 3$ and $\mathrm{p}-\mathrm{HA} 2 \mathrm{X}$ and cleaved PARP as expected. However, treating shTACC 3 cells with BO-264 did not further induce mitotic arrest, DNA damage, and apoptosis compared with untreated shTACC3 cells, further supporting the specificity of our TACC3 inhibitor, BO-264. Overall, these results demonstrate that the observed sensitivity of breast cancer cell line models to $\mathrm{BO}-264$ is potentially due to a stronger induction of the downstream mitotic cell death pathways upon specific TACC3 inhibition with BO-264 treatment.

We then tested a potential disruption of mitotic spindles as TACC 3 inhibition has previously been demonstrated to cause severe spindle defects (9). Inhibition of TACC 3 with BO-264 has led to formation of aberrant spindle structures in a dose-dependent manner (Fig. 5D). The most prominent phenotype seen upon TACC3 inhibition by BO264 was the formation of multipolar, disorganized, and monopolar spindles characterized by improperly aligned chromosomes at the metaphase plate $(73.4 \%, 24.5 \%$, and $1.5 \%$ frequency, respectively, upon treatment with the highest dose) in JIMT-1 cells (Fig. 5E). TACC3 knockdown with two different siRNAs recapitulated the effects of BO-264. However, treatment of siTACC3 cells with BO264 did not further increase the number of aberrant spindles compared with vehicle-treated siTACC3 cells (Supplementary Fig. S4F and S4G), further supporting the on-target effect of BO-264. The presence of spindle defects activates the SAC that further leads to mitotic arrest to provide the cells with necessary time to repair spindle defects (38). To demonstrate SAC activation upon TACC3 inhibition, we performed immunofluorescence staining of BuBR1, a marker of SAC activation (39), in BO-264-treated JIMT-1 cells and observed an increased localization of BuBR1 to chromosomes upon BO-264 treatment (Fig. 5F). To further test the contribution of an active SAC signaling to BO-264-induced mitotic arrest, DNA damage, and cell death, we inhibited the SAC kinase, Mps1 using a specific inhibitor (TC Mps1; ref. 40) in BO-264-treated cells and analyzed the expression of relevant markers. As shown in Fig. 5G, inhibiting Mps1 kinase caused a prominent loss of mitotic arrest, apoptosis, and DNA damage suggesting that BO-264 may function by activating SAC upon inducing severe spindle defects that further leads to prolonged mitosis, apoptotic cell death, and DNA damage. TACC3 has also been shown to localize to the centrosomes during mitosis (7) and interphase (13). To test whether BO-264 alters TACC3 localization in JIMT-1 cells, we performed immunofluorescence staining of TACC3 together with a centrosome marker, $\gamma$-tubulin $($ Fig. $\mathbf{5 H}$ ). We observed that centrosomal localization of TACC3 during mitosis was greatly reduced upon BO-264 treatment (Fig. 5I). We also observed a significant decrease in TACC 3 localization in centrosomes during interphase cells, suggesting loss of microtubule nucleation from centrosomes upon TACC3 inhibition (Fig. 5J and $\mathbf{K})$. These results clearly state that BO-264 alters intracellular localization of TACC3 and its cellular function.

\section{BO-264 significantly impairs tumor growth and improves survival without major toxicity}

To further test the antitumorigenic potential of TACC 3 inhibition by BO-264 in the in vivo settings, we utilized the xenografts of HER2+ JIMT-1 cell line that is known to be highly tumorigenic (41), and that we demonstrated the expression of high levels of TACC3 (Supplementary Fig. S3A) along with the high sensitivity to BO-264 (Fig. 3A). To this end, JIMT-1 cells were injected into mammary fat pad (MFP) of female nude mice, and mice were subsequently treated with vehicle or BO-264 (25 mg/kg) for 3-4 weeks. BO-264-treated mice showed a significant suppression of tumor growth compared with vehicle-treated mice (Fig. 6A). Moreover, BO-264 was welltolerated because treatment did not cause a significant body weight loss (Fig. 6B) and organ toxicity (Fig. 6C). We further tested the effect of TACC3 inhibition in vivo using an immunocompetent mouse model. Murine triple-negative breast cancer cell line, EMT6, which is a fast-growing and highly aggressive model (42) with high TACC3 level compared with normal mouse embryonic fibroblast cell line (Supplementary Fig. S5A), was injected into MFPs of syngeneic $\mathrm{Balb} / \mathrm{c}$ mice. We observed a significant tumor growth inhibition and $57.1 \%$ increased lifespan in BO-264-treated mice compared with vehicle-treated ones (Fig. 6D and E). Moreover, BO-264 was again well-tolerated in the syngeneic mouse model as shown with body weight change (Fig. 6F) and organ hematoxylin and eosin (H\&E) staining (Fig. 6G).

As TACC3 deregulation is observed also in colon cancer and its level play an important role for disease progression (43), and we showed that colon cancer cell lines in NCI-60 panel are very sensitive to BO-264 (Fig. 4E), we aimed to test the effect of TACC3 inhibition with BO-264 in colon cancer models. Analyses of TCGA and GSE39582 confirmed the overexpression of TACC 3 in colon cancer tissues compared with normal tissues (Supplementary Fig. S5B and S5C). Before testing the effect of BO-264 on tumor growth of colon cancer models, we first analyzed TACC 3 protein levels in colon cancer cell lines and found that HCT-116 (human colorectal carcinoma) and CT-26 (murine colon carcinoma) have high TACC3 protein levels (Supplementary Fig. S5A). We also showed that inhibition of TACC3 with BO-264 leads to mitotic arrest, DNA damage, and apoptosis, similar to the results we obtained in breast cancer (Supplementary Fig. S5D). Finally, we tested antitumorigenic effect of BO-264 $(50 \mathrm{mg} / \mathrm{kg})$ on colon cancer xenografts (HCT-116) and syngeneic (CT-26) tumor models and demonstrated that BO-264 significantly impairs tumor growth (Supplementary Fig. S5E) without any significant toxicity (Supplementary Fig. S5F) in both models. Altogether, these data show that BO-264 is effective not only for breast cancer, but also for colon cancer in vivo.

Finally, to further compare the antitumorigenic effects of BO-264 and SPL-B in the in vivo settings, we treated JIMT-1 xenografts with BO-264 and SPL-B, side by side. As shown in Supplementary Fig. S6A and $\mathrm{S} 6 \mathrm{~B}$, while $5 \mathrm{mg} / \mathrm{kg}$ dosing of SPL-B failed to prevent tumor growth, BO-264 was significantly more effective in delaying tumor growth with no major toxicity that further supports the superior anticancer activity of BO-264. Furthermore, when $5 \mathrm{mg} / \mathrm{kg}$ (Supplementary Fig. S6A) and $25 \mathrm{mg} / \mathrm{kg}$ (Fig. 6A) data of BO-264 were compared, we see a clear dose-dependent effect on tumor growth. These results indicate that oral administration of BO-264 significantly inhibits tumor growth and improves survival in mice models of aggressive breast cancer. 
A

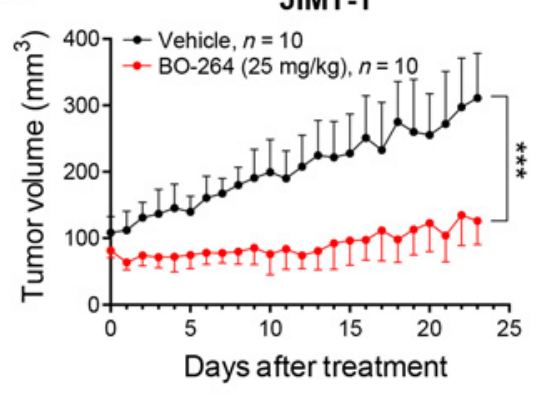

B

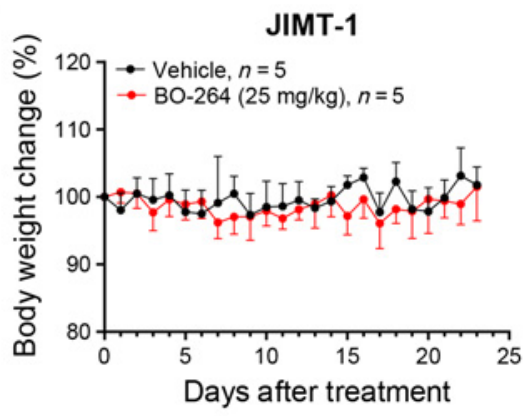

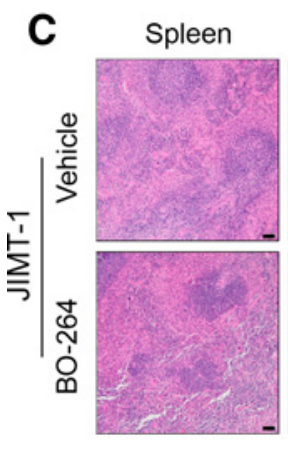
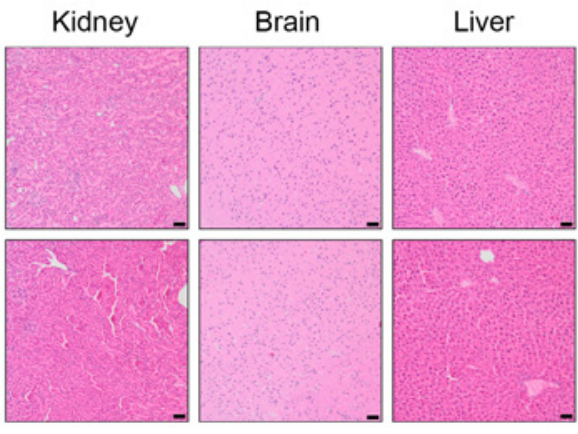

Lung

D

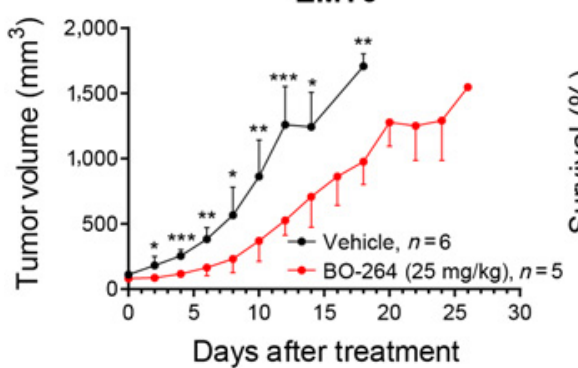

E

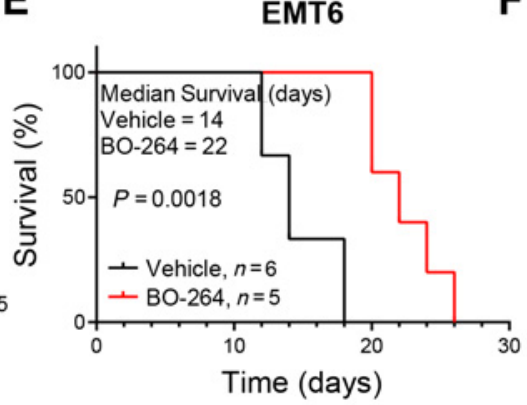

$\mathbf{F}$

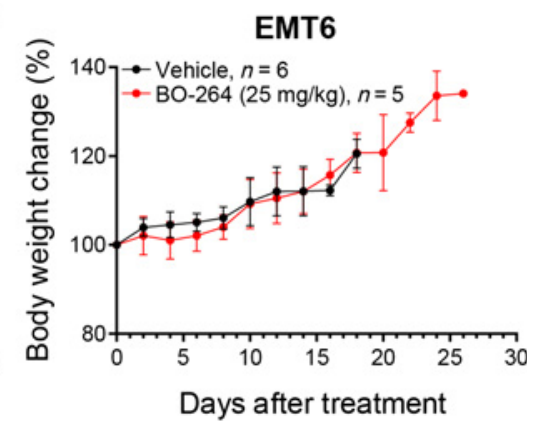

G
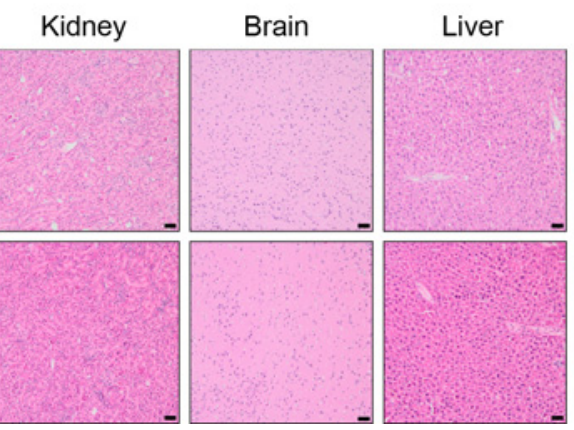

Lung

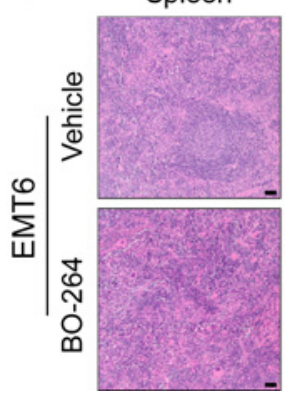

Figure 6.

BO-264 significantly impairs tumor growth and improves survival in in vivo xenograft and syngeneic models without major toxicity. A, Tumor volume change of JIMT-1 xenografts after the treatment with vehicle or $25 \mathrm{mg} / \mathrm{kg} \mathrm{BO}^{-264}$ in female nude mice. Treatments were started when tumors reached $90-100$ mm ${ }^{3}$. B, Body weight change (\%) of nude mice bearing JIMT-1 xenografts. C, Histologic analysis of major organs (spleen, kidney, brain, liver, and lungs) of JIMT-1 xenografts by H\&E staining. Representative images for each organ are presented. Scale bar, $50 \mu \mathrm{m}$. D, Tumor volume measurements of the effect of BO-264 on a highly aggressive breast cancer mouse model with EMT6 cells. Significance was calculated using multiple $t$ test for each data point for each day compared with vehicle control. E, Kaplan-Meier survival curve of EMT6 syngeneic tumors treated with vehicle (median survival 14 days) or BO-264 (median survival 22 days). $P$ value was calculated using the logrank test. ${ }^{*}, P<0.05 ;{ }^{* *}, P<0.01 ;{ }^{* * *}, P<0.001$. F, Body weight change (\%) of Balb/c mice bearing EMT6 syngeneic tumors. $\mathbf{G}$, H\&E staining of major organs in EMT6 syngeneic model. Representative images of for each organ are presented. Scale bar, $50 \mu \mathrm{m}$. 


\section{Discussion}

TACC3, an oncogenic member of the TACC family of microtubule- and centrosome-binding proteins, emerges as an attractive anticancer target that is strongly associated with worse prognosis in various different cancers (15-18). Our in-house screening efforts with a series of small-molecule compounds in a cytotoxicity assay supported by different biochemical and functional assays led to the identification of BO-264 as a novel inhibitor of the TACC3 protein. We demonstrated that treatment of aggressive breast cancer cell lines with BO-264 induces the formation of multipolar spindles that activates SAC and decreases centrosomal localization of TACC3. This, in turn, leads to mitotic arrest followed by apoptotic cell death at very low doses as compared with other TACC3 inhibitors. These results phenocopied the siRNA-mediated knockdowns of TACC3, demonstrating the on-target specificity of our novel inhibitor. This specificity is further supported by shRNA- and siRNA-mediated knockdowns of TACC3 where BO-264 was not able to induce mitotic arrest, DNA damage, and apoptosis, and cells with aberrant spindles, respectively, in the absence of TACC3. Oral administration of BO-264 to immunocompetent or immunodeficient mouse models of aggressive breast and colon cancer resulted in a significant tumor growth inhibition and improved survival of tumorbearing mice with no major toxicity, providing a strong preclinical support for targeting TACC3 in aggressive cancers.

MTAs that interfere with microtubule dynamics is one of the most effective group of chemotherapeutics and are widely used in different clinical settings (44). However, due to lack of selectivity for cancer cells, MTAs also cause cell death in normal cells and thus lead to severe side effects. Moreover, patients frequently develop resistance to MTAs that limits their long-term clinical use (45). In this line, TACC3-targeting agents have a potential to emerge as cancer-specific microtubule inhibitors that might function in a similar fashion with MTAs in terms of the antitumorigenic effects, but are expected to cause less side effects owing to the lack of a strong expression of TACC3 protein in normal tissues. In this line, BO-264 shows promising antitumor effect with no major toxicity in both breast and colon cancer models. However, future studies are needed to test the inhibitor at a wider dose range to assess its tolerability and determine the therapeutic window. Furthermore, in vivo pharmacokinetic analyses are required to determine the in vivo accessibility and distribution of our hit compound that can further be optimized to achieve a better tumor growth inhibition.

We demonstrated a strong overexpression of TACC3 in tumors from different cancers as compared with their normal counterparts using the TCGA and other published data, as well as in breast cancer cell lines as compared with a normal breast cell line, MCF-12A. We further showed enhanced survival upon TACC3 overexpression in MCF-12A cells, which have very low basal levels of TACC3. Importantly, expressing TACC3 in these cells rendered them sensitive to growth inhibition by BO-264 that further validates the specificity of our inhibitor. Furthermore, we detected a trend toward increased expression of TACC3 in basal and HER2 + subtypes, which are more aggressive breast cancer subtypes compared with luminal A subtype, and they were more sensitive to TACC3 inhibition with BO-264. Overall, these results suggest that TACC3 expression might be a determinant (but not the sole determinant) of BO-264 response, which might further help in stratifying patients that will most benefit from TACC3 inhibition by BO-264.

We showed that BO-264 effectively inhibits the growth of cancer cell lines, harboring the highly oncogenic FGFR3-TACC3 fusion protein. This fusion protein has been detected in a variety of different solid tumors and was shown to drive tumorigenesis by stimulating constitutive activation of the FGFR signaling (30). Recently, Balversa (erdafitinib), an FGFR kinase inhibitor, has been approved for patients with metastatic urothelial carcinoma with FGFR alterations, including FGFR3-TACC3 fusion (46). However, response rates to FGFR inhibition are still limited (around 36\%; ref. 46), suggesting that TACC3 targeting alone or in combination with FGFR inhibition might provide a superior anticancer activity in models with FGFR3-TACC3 fusion. We demonstrated that $\mathrm{BO}-264$ inhibits proliferation in two different fusion-carrying models, to different extend although, depending on TACC3 levels, suggesting that our inhibitor might represent a novel strategy to combat FGFR3-TACC3 fusion-carrying cancers.

We demonstrated a strong, dose-dependent induction of multipolar spindles and reduction in centrosomal localization of TACC3 upon BO-264 treatment, suggesting that BO-264 strongly affects the centrosomal functions of the TACC3 protein. Centrosome amplifications are frequently observed in cancers and are strongly associated with cancer aggressiveness (47). Clustering extra centrosomes into two spindle poles during mitotic cell division is vital for cancer cells with supernumerary centrosomes to undertake a bipolar cell division and prevent aneuploidy (48). Therapeutic strategies that target centrosome clustering have been suggested to be highly effective in mediating specific cell killing in the highly aggressive, centrosome-amplified tumors (49). TACC3 has previously been shown to trigger centrosome clustering downstream of the integrin-linked kinase, which aids in the Aurora A-mediated phosphorylation of TACC3 (8). Our data showing a strong induction of multipolar spindles and centrosome localization upon BO-264 treatment in JIMT-1 cells, which harbors amplified centrosomes (50), further corroborates the importance of TACC3 for centrosome integrity and suggest that it might be a highly relevant target in centrosome-amplified cancers, necessitating further research.

Overall, we identified a novel TACC3 inhibitor that effectively kills cancer cells at comparably lower doses than other available TACC3 inhibitors both in vitro and in vivo. We validated the specific binding of BO-264 to TACC3 protein using different biochemical assays. We further tested the specificity by comparing BO-264-induced molecular and phenotypic changes with those mediated by siRNA- and ShRNAmediated knockdowns. We demonstrated that pharmacologic inhibition of TACC3 with BO-264 fully recapitulates genomic knockdown in terms of the activation of SAC, induction of mitotic arrest, apoptosis, and DNA damage. We further identified the formation of multipolar spindles, indicative of a potential disintegration of centrosomes upon BO-264 treatment that might further be investigated to explore the centrosomal functions of TACC3 in more detail. Finally, the strong antitumorigenic effect that we observed in two different aggressive breast and colon cancer in vivo models without any apparent organ toxicity provides a strong preclinical support for developing this inhibitor further for future clinical tests to treat breast, colon, and potentially other cancers.

\section{Disclosure of Potential Conflicts of Interest}

Erden Banoglu, Ozgur Sahin and Burcu Caliskan are both applicants and inventors in patent application (patent number: PCT/TR2019/050951 and EP19209120.5) entitled "Isoxazole Derivatives Targeting TACC3 as Anticancer Agents" while Deniz Lengerli and Ozge Akbulut are inventors. No potential conflicts of interest were disclosed by the other authors.

\section{Authors' Contributions}

Conception and design: B. Caliskan, E. Banoglu, O. Sahin Development of methodology: O. Akbulut, D. Lengerli, U.O.S. Seker Acquisition of data (provided animals, acquired and managed patients, provided facilities, etc.): O. Akbulut, O. Saatci, E. Duman, A. Akyol 
Analysis and interpretation of data (e.g., statistical analysis, biostatistics, computational analysis): O. Akbulut, D. Lengerli, O. Saatci, E. Duman, A. Isik, B. Caliskan, E. Banoglu, O. Sahin

Writing, review, and/or revision of the manuscript: O. Akbulut, O. Saatci, B. Caliskan, E. Banoglu, O. Sahin

Study supervision: B. Caliskan, E. Banoglu, O. Sahin

Other (synthesis of the compound BO264): D. Lengerli

\section{Acknowledgments}

We thank the NCI Developmental Therapeutics Program for screening the anticancer activity of BO-264 in NCI-60 human tumor cell lines. We thank
Prof. Dr. Mesut Muyan (Middle East Technical University, Ankara, Turkey) for providing the pCDNA3.1(+) vector. We also thank METABRIC Consortium for providing us with breast cancer data. This study was, in part, supported by European Molecular Biology Organization Installation Grant 2791 (to O. Sahin).

The costs of publication of this article were defrayed in part by the payment of page charges. This article must therefore be hereby marked advertisement in accordance with 18 U.S.C. Section 1734 solely to indicate this fact.

Received October 5, 2019; revised February 11, 2020; accepted March 19, 2020; published first March 26, 2020.

\section{References}

1. Zhou J, Giannakakou P. Targeting microtubules for cancer chemotherapy Curr Med Chem Anticancer Agents 2005;5:65-71.

2. Tangutur AD, Kumar D, Krishna KV, Kantevari S. Microtubule targeting agents as cancer chemotherapeutics: an overview of molecular hybrids as stabilizing and destabilizing agents. Curr Top Med Chem 2017;17:2523-37

3. Wozniak KM, Vornov JJ, Wu Y, Liu Y, Carozzi VA, Rodriguez-Menendez V, et al. Peripheral neuropathy induced by microtubule-targeted chemotherapies: insights into acute injury and long-term recovery. Cancer Res 2018;78: 817-29.

4. Ganguly A, Cabral F. New insights into mechanisms of resistance to microtubule inhibitors. Biochim Biophys Acta 2011;1816:164-71.

5. Strebhardt K. Ullrich A, et al. Aurora kinases: novel therapy targets in cancers. Oncotarget 2017;8:23937-54.

6. Gergely F, Karlsson C, Still I, Cowell J, Kilmartin J, Raff JW. The TACC domain identifies a family of centrosomal proteins that can interact with microtubules. Proc Natl Acad Sci U S A 2000;97:14352-7.

7. Nwagbara BU, Faris AE, Bearce EA, Erdogan B, Ebbert PT, Evans MF, et al TACC3 is a microtubule plus end-tracking protein that promotes axon elongation and also regulates microtubule plus end dynamics in multiple embryonic cell types. Mol Biol Cell 2014;25:3350-62.

8. Fielding AB, Lim S, Montgomery K, Dobreva I, Dedhar S. A critical role of integrin-linked kinase, ch-TOG and TACC 3 in centrosome clustering in cancer cells. Oncogene 2011;30:521-34.

9. Schneider L, Essmann F, Kletke A, Rio P, Hanenberg H, Wetzel W, et al. The transforming acidic coiled coil 3 protein is essential for spindledependent chromosome alignment and mitotic survival. J Biol Chem 2007;282:29273-83.

10. Petschnigg J, Kotlyar M, Blair L, Jurisica I, Stagljar I, Ketteler R. Systematic identification of oncogenic EGFR interaction partners. J Mol Biol 2017;429: 280-94.

11. Ha GH, Kim JL, Breuer EK. TACC3 is essential for EGF-mediated EMT in cervical cancer. PLoS One 2013;8:e70353.

12. Schneider L, Essmann F, Kletke A, Rio P, Hanenberg H, Schulze-Osthoff K, et al TACC3 depletion sensitizes to paclitaxel-induced cell death and overrides p21WAF-mediated cell cycle arrest. Oncogene 2008;27:116-25.

13. Foraker AB, Camus SM, Evans TM, Majeed SR, Chen CY, Taner SB, et al Clathrin promotes centrosome integrity in early mitosis through stabilization of centrosomal ch-TOG. J Cell Biol 2012;198:591-605.

14. Fu W, Jiang $\mathrm{Q}$, et al. Novel functions of endocytic player clathrin in mitosis. Cell Res 2011;21:1655-61.

15. Li Q, Ye L, Guo W, Wang M, Huang S, Peng X. Overexpression of TACC3 is correlated with tumor aggressiveness and poor prognosis in prostate cancer. Biochem Biophys Res Commun 2017;486:872-8.

16. Zhou DS, Wang HB, Zhou ZG, Zhang YJ, Zhong Q, Xu L, et al. TACC3 promotes stemness and is a potential therapeutic target in hepatocellular carcinoma. Oncotarget 2015;6:24163-77.

17. Jung CK, Jung JH, Park GS, Lee A, Kang CS, Lee KY. Expression of transforming acidic coiled-coil containing protein 3 is a novel independent prognostic marker in non-small cell lung cancer. Pathol Int 2006;56:503-9.

18. Song H, Liu C, Shen N, Yi P, Dong F, Li X, et al. Overexpression of TACC3 in breast cancer associates with poor prognosis. Appl Immunohistochem $\mathrm{Mol}$ Morphol 2018;26:113-9.

19. Wurdak H, Zhu S, Min KH, Aimone L, Lairson LL, Watson J, et al. A small molecule accelerates neuronal differentiation in the adult rat. Proc Natl Acad Sci U S A 2010;107:16542-7.

20. Polson ES, Kuchler VB, Abbosh C, Ross EM, Mathew RK, Beard HA, et al. KHS101 disrupts energy metabolism in human glioblastoma cells and reduces tumor growth in mice. Sci Transl Med 2018;10:pii: eaar2718.

21. Campo L, Breuer EK. Inhibition of TACC3 by a small molecule inhibitor in breast cancer. Biochem Biophys Res Commun 2018;498:1085-92.

22. Guo F, Liu Y. Knockdown of TACC 3 inhibits the proliferation and invasion of human renal cell carcinoma cells. Oncol Res 2018;26:183-9.

23. Pai MY, Lomenick B, Hwang H, Schiestl R, McBride W, Loo JA, et al. Drug affinity responsive target stability (DARTS) for small-molecule target identification. Methods Mol Biol 2015;1263:287-98.

24. Martinez Molina D, Jafari R, Ignatushchenko M, Seki T, Larsson EA, et al. Monitoring drug target engagement in cells and tissues using the cellular thermal shift assay. Science 2013;341:84-7.

25. Nagy A, Lanczky A, Menyhart O, Gyorffy B. Validation of miRNA prognostic power in hepatocellular carcinoma using expression data of independent datasets. Sci Rep 2018;8:9227.

26. Curtis C, Shah SP, Chin SF, Turashvili G, Rueda OM, Dunning MJ, et al. The genomic and transcriptomic architecture of 2,000 breast tumours reveals novel subgroups. Nature 2012;486:346-52.

27. Yao R, Kondoh Y, Natsume Y, Yamanaka H, Inoue M, Toki H, et al. A small compound targeting TACC3 revealed its different spatiotemporal contributions for spindle assembly in cancer cells. Oncogene 2014;33:4242-52.

28. Lomenick B, Jung G, Wohlschlegel JA, Huang J. Target identification using drug affinity responsive target stability (DARTS). Curr Protoc Chem Biol 2011;3:163-80.

29. Pierce MM, Raman CS, Nall BT. Isothermal titration calorimetry of proteinprotein interactions. Methods 1999;19:213-21.

30. Costa R, Carneiro BA, Taxter T, Tavora FA, Kalyan A, Pai SA, et al. FGFR3TACC3 fusion in solid tumors: mini review. Oncotarget 2016;7:55924-38.

31. Williams SV, Hurst CD, Knowles MA. Oncogenic FGFR3 gene fusions in bladder cancer. Hum Mol Genet 2013;22:795-803.

32. McFarland JM, Ho ZV, Kugener G, Dempster JM, Montgomery PG, Bryan JG, et al. Improved estimation of cancer dependencies from large-scale RNAi screens using model-based normalization and data integration. Nat Commun 2018;9: 4610 .

33. Kimura M, Yoshioka T, Saio M, Banno Y, Nagaoka H, Okano Y. Mitotic catastrophe and cell death induced by depletion of centrosomal proteins. Cell Death Dis 2013;4:e603.

34. Saatci O, Borgoni S, Akbulut O, Durmus S, Raza U, Eyupoglu E, et al. Targeting PLK1 overcomes T-DM1 resistance via CDK1-dependent phosphorylation and inactivation of $\mathrm{Bcl}-2 / \mathrm{xL}$ in HER2-positive breast cancer. Oncogene 2018;37: 2251-69.

35. Prigent C, Dimitrov S. Phosphorylation of serine 10 in histone $\mathrm{H} 3$, what for? J Cell Sci 2003;116:3677-85.

36. Chaitanya GV, Steven AJ, Babu PP. PARP-1 cleavage fragments: signatures of cell-death proteases in neurodegeneration. Cell Commun Signal 2010;8:31.

37. Sharma A, Singh K, Almasan A. Histone H2AX phosphorylation: a marker for DNA damage. Methods Mol Biol 2012;920:613-26.

38. Musacchio A, Salmon ED. The spindle-assembly checkpoint in space and time. Nat Rev Mol Cell Biol 2007;8:379-93.

39. Chen RH. BubR1 is essential for kinetochore localization of other spindle checkpoint proteins and its phosphorylation requires Mad1. J Cell Biol 2002; 158:487-96.

40. Choi M, Min YH, Pyo J, Lee CW, Jang CY, Kim JE, et al. a novel Mps1 inhibitor, suppresses the growth of hepatocellular carcinoma cells via the accumulation of chromosomal instability. Br J Pharmacol 2017;174:1810-25. 
41. Tanner M, Kapanen AI, Junttila T, Raheem O, Grenman S, Elo J, et al Characterization of a novel cell line established from a patient with Herceptin-resistant breast cancer. Mol Cancer Ther 2004;3:1585-92.

42. Yang Y, Yang HH, Hu Y, Watson PH, Liu H, Geiger TR, et al. Immunocompetent mouse allograft models for development of therapies to target breast cancer metastasis. Oncotarget 2017;8:30621-43.

43. Du Y, Liu L, Wang C, Kuang B, Yan S, Zhou A, et al. TACC3 promotes colorectal cancer tumourigenesis and correlates with poor prognosis. Oncotarget 2016;7: 41885-97.

44. Bates D, Eastman A. Microtubule destabilising agents: far more than just antimitotic anticancer drugs. Br J Clin Pharmacol 2017;83 255-68.

45. Yardley DA. Drug resistance and the role of combination chemotherapy in improving patient outcomes. Int J Breast Cancer 2013;2013: 137414.
46. Loriot Y, Necchi A, Park SH, Garcia-Donas J, Huddart R, Burgess E, et al. Erdafitinib in locally advanced or metastatic urothelial carcinoma. N Engl J Med 2019;381:338-48

47. Godinho SA, Picone R, Burute M, Dagher R, Su Y, Leung CT, et al. Oncogene-like induction of cellular invasion from centrosome amplification. Nature 2014;510:167-71.

48. Kwon M, Godinho SA, Chandhok NS, Ganem NJ, Azioune A, Thery M, et al. Mechanisms to suppress multipolar divisions in cancer cells with extra centrosomes. Genes Dev 2008;22:2189-203.

49. Sabat-Pospiech D, Fabian-Kolpanowicz K, Prior IA, Coulson JM, Fielding AB. Targeting centrosome amplification, an Achilles' heel of cancer. Biochem Soc Trans 2019;47:1209-22.

50. Arnandis T, Monteiro P, Adams SD, Bridgeman VL, Rajeeve V, Gadaleta E, et al Oxidative stress in cells with extra centrosomes drives non-cell-autonomous invasion. Dev Cell 2018;47:409-24. 


\title{
Molecular Cancer Therapeutics
}

\section{A Highly Potent TACC3 Inhibitor as a Novel Anticancer Drug Candidate}

Ozge Akbulut, Deniz Lengerli, Ozge Saatci, et al.

Mol Cancer Ther 2020;19:1243-1254. Published OnlineFirst March 26, 2020.

\author{
Updated version Access the most recent version of this article at: \\ doi:10.1158/1535-7163.MCT-19-0957 \\ Supplementary Access the most recent supplemental material at: \\ Material http://mct.aacrjournals.org/content/suppl/2020/03/26/1535-7163.MCT-19-0957.DC1
}

Cited articles This article cites 50 articles, 12 of which you can access for free at:

http://mct.aacrjournals.org/content/19/6/1243.full\#ref-list-1

E-mail alerts Sign up to receive free email-alerts related to this article or journal.

Reprints and

To order reprints of this article or to subscribe to the journal, contact the AACR Publications Department a Subscriptions pubs@aacr.org.

Permissions To request permission to re-use all or part of this article, use this link http://mct.aacrjournals.org/content/19/6/1243.

Click on "Request Permissions" which will take you to the Copyright Clearance Center's (CCC)

Rightslink site. 